\title{
TECHNOLOGY ADVANCES
}

\section{Graphite Foam with High Thermal Conductivity and Diffusivity Conducts Heat in All Directions}

Most foamed materials serve as good thermal insulators because of their highly porous nature. Carbon foam materials, which have been produced for several years, are composed of amorphous carbon and have densities ranging from 0.12 to $0.50 \mathrm{~g} / \mathrm{cm}^{3}$. Their amorphous structure makes them good thermal insulators with thermal conductivities less than $10 \mathrm{~W} / \mathrm{m}$ K. Scientists at Oak Ridge National Laboratories have developed a graphitic carbon foam with exceptionally high thermal conductivity and efficient energy-transfer characteristics. This patented technology is now being commercialized by Poco Graphite Inc. (POCO) as PocoFoam ${ }^{\mathrm{TM}}$.

PocoFoam is derived from mesophase pitch, an intermediate phase in the formation of carbon from pitch that when heated above $2000^{\circ} \mathrm{C}$ forms graphite. This precursor material, combined with an innovative production method, produces a material with very high thermal conductivity. It differs from conventional carbon foams in that the ligaments making up the honeycomb-like structure of the foam (see Figure 1) are of a highly aligned, graphitic nature rather than an amorphous one. This difference in crystallinity gives PocoFoam its useful characteristics that include dimensional stability, a low coefficient of thermal expansion, relatively high modulus of elasticity and compressive strength, and very high thermal conductivity (see Table I).

The overall thermal conductivity of PocoFoam is comparable to metals such as copper or aluminum currently used in many thermal-management applications. Its density, however, is only about $6 \%$ that of copper and about $20 \%$ that of aluminum. With respect to specific thermal

Technology Advances provides upto-date reports of materials developments that show potential to bridge the gap between research innovation and application of advanced materials technologies. If you encounter or are involved with materials research that shows potential for commercialization and would like to present these developments, contact Renée G. Ford, Renford Communications, Ltd., P.O. Box 72, Harrison, NY 10528-0072; tel. 914-967-0955; fax 914-967-7927; or e-mail renford@compuserve.com.

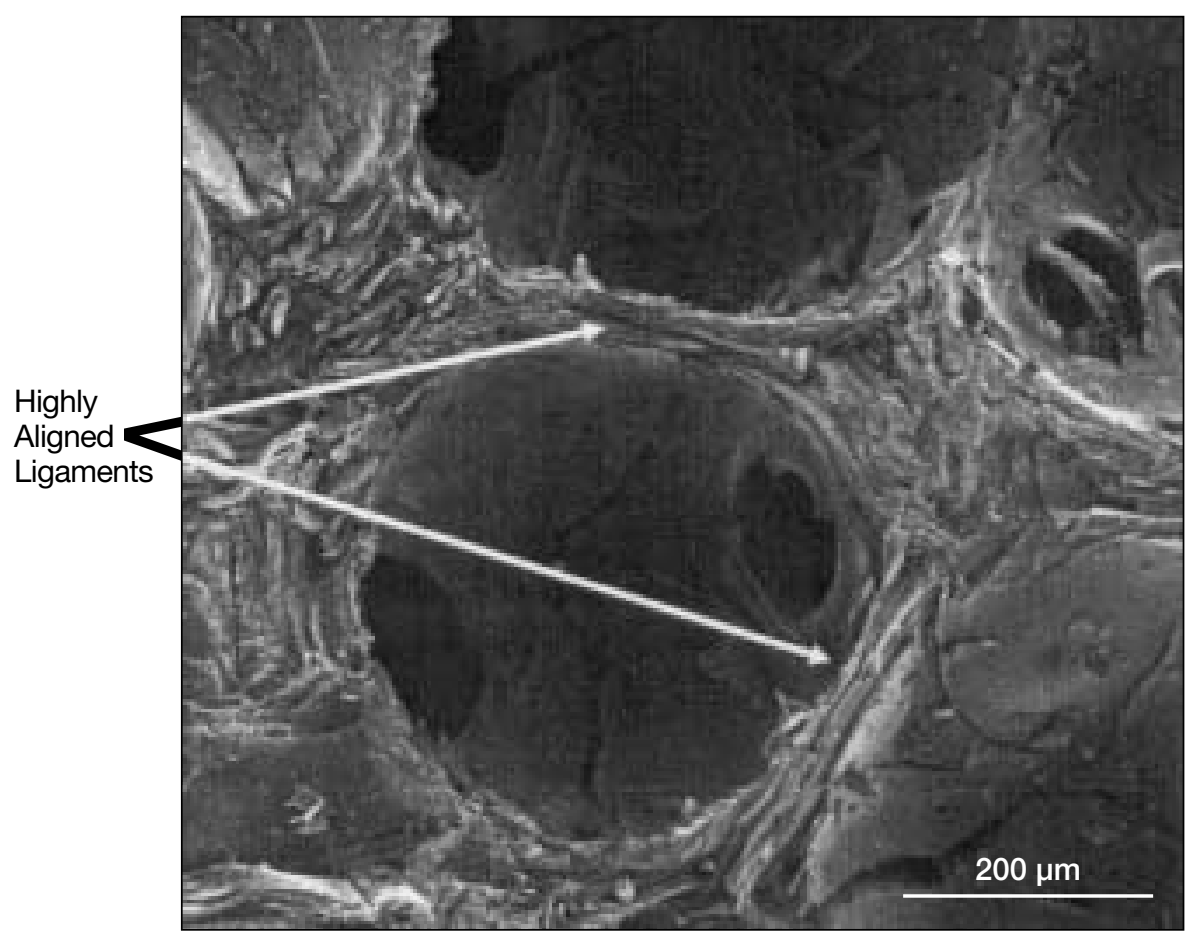

Figure 1. Photomicrograph of the highly aligned, graphitic ligaments of PocoFoam ${ }^{\mathrm{TM}}$.

conductivity (overall thermal conductivity divided by specific gravity), it outperforms these materials (see Table II), which is an advantageous property for fabricating lightweight heat-transfer devices. PocoFoam's significant advantage, compared with other materials that also have high thermal conductivities such as carbon-carbon composites and graphite fibers, is that it conducts heat in all directions. Composites and fibers only conduct well in the direction of the fiber.

Another useful thermal property of PocoFoam is its thermal diffusivity, which is a measure of how quickly heat is transported through a material compared with how quickly the material absorbs the heat. Thermal diffusivity is defined as thermal conductivity divided by the product of density and specific heat. PocoFoam's high thermal conductivity, coupled with low density and low specific heat, results in high thermal diffusivity. This is evidenced by heating very uniformly and quickly throughout its bulk.

More than $96 \%$ of PocoFoam's porosity is interconnected or open porosity. This gives it a very high internal surface area and, consequently, efficient heat transfer to any working fluid passing through it. Its low density, open porosity, and high thermal conductivity make it a promising material for thermal management in
Table I: Typical Properties of PocoFoam ${ }^{\mathrm{TM}}$

\section{Mechanical Properties}

Apparent Density

Total Porosity

Open Porosity

$0.2-0.6 \mathrm{~g} / \mathrm{cm}^{3}$

Pore Diameter

Specific Surface Area

Modulus of Elasticity

Compressive Strength

$73-82 \%$

$>96 \%$

$350 \mu \mathrm{m}$

$>4 \mathrm{~m}^{2} / \mathrm{g}$

$0.04 \mathrm{GPa}$

$3.4 \mathrm{MPa}$ (at $0.6 \mathrm{~g} / \mathrm{cm}^{3}$ )

\section{Thermal Properties}

Thermal Conductivity

Specific Thermal

Conductivity

Coefficient of

Thermal Expansion

Specific Heat

$100-150 \mathrm{~W} / \mathrm{m} \mathrm{K}$

$179-268 \mathrm{~W} / \mathrm{m} \mathrm{K}$

$2.0 \mu \mathrm{m} / \mathrm{m} \mathrm{K}$

$0.7 \mathrm{~J} / \mathrm{kg} \mathrm{K}$

applications where high efficiency and low mass are critical. Heat sinks for laptop computers, radiators for race cars, and heat exchangers for satellites are a few of the applications currently being explored.

The company is currently investigating various processes to enhance the finished product, such as chemical vapor infiltration (CVI) with carbon to increase rigidity and strength, coating a surface with metal to provide a thermally conductive attachment through brazing, and purification to 
Table II: Comparison of Thermal Conductivity and Specific Thermal Conductivity

\begin{tabular}{lccccc}
\hline & \multicolumn{2}{c}{ Thermal Conductivity } & \multicolumn{2}{c}{$\begin{array}{c}\text { Specific Thermal } \\
\text { Conductivity* }\end{array}$} \\
\hline Material & $\begin{array}{c}\text { Specific } \\
\text { Gravity }\end{array}$ & $\begin{array}{c}\text { With Grain } \\
\text { W/m K }\end{array}$ & $\begin{array}{c}\text { Across Grain } \\
\text { W/m K }\end{array}$ & $\begin{array}{c}\text { With Grain } \\
\text { W/m K }\end{array}$ & $\begin{array}{c}\text { Across Grain } \\
\text { W/m K }\end{array}$ \\
\hline PocoFoam & 0.56 & & 150 & & 268 \\
Copper & 8.9 & & 400 & & 45 \\
Aluminum 6061 & 2.8 & & 180 & & 64 \\
EWC-300 Epoxy Resin & 1.72 & 109 & 1 & 63 & 0.6 \\
K321/AR Pitch & 1.77 & 233 & 20 & 132 & 11 \\
Carbon-Carbon & 1.76 & 650 & 20 & 369 & 11 \\
Amoco SRG (Carbon fiber) & 0.5 & & 12 & & 24 \\
Aluminum Foam & 0.5 & & & & \\
\hline
\end{tabular}

*Specific thermal conductivity is defined as thermal conductivity divided by specific gravity. reduce impurity levels to less than 9 ppm. Two process patents have been issued for the production method, and several others are pending. Pilot production runs have been completed, and full-scale production is anticipated by the end of this year.

\section{Opportunities}

POCO is interested in establishing relationships with industrial partners to develop PocoFoam for specific applications.

Source: Lee Wiechmann, PocoFoam Group, POCO Graphite Inc., 1601 S. State Street, Decatur, TX 76234, USA; tel. 940-393-4462; fax 940-393-8435; e-mail foam@poco.com; www.pocofoam.com.

\section{Electrochemical Method Uses Molten Calcium Chloride to Extract Metals Directly from their Oxides}

Metal oxides are usually reduced by carbon, hydrogen, a metal with a more stable oxide than the oxide to be reduced, or by dissolving the oxide in either an aqueous or fused salt solution and electrolyzing the solution. Scientists at the Department of Materials Science, University of Cambridge, have discovered yet another way of reducing metal oxides: In their approach, the cathode in the electrolytic cell, a solid oxide, is in a melt of calcium chloride at about $900^{\circ} \mathrm{C}$, and a current is passed through it. The researchers have found that the favored cathodic reaction is the ionization of oxygen, $\mathrm{O}+2 \mathrm{e}^{-}=\mathrm{O}^{2-}$ from the metal oxide and not the deposition of calcium, $\mathrm{Ca}^{2+}+$ $2 \mathrm{e}^{-}=\mathrm{Ca}$. The oxygen ions readily dissolve in the calcium chloride electrolyte and diffuse to the anode, where they are discharged. Calcium chloride has several desirable properties: a very high solubility for oxygen ions, a high solubility in water, a very low toxicity, and ready availability.

The overall process for extracting titanium from titanium dioxide is the removal of oxygen from the titanium dioxide, leaving titanium metal behind. It is a far simpler process than the established route for extracting titanium that was discovered by William Kroll in 1940. His process consists of several complicated stepscabochlorination of titanium dioxide to form titanium tetrachloride, reduction of the tetrachloride by magnesium, separation of the titanium from the magnesium chloride by distillation, and finally, the electrolysis of the magnesium chloride to give chlorine and magnesium for recycling back into the processes. There are many more steps than in most pyrometallurgical processes, and the size of the reactors is small-each reactor can only produce about 7 tons per week. In most other metallurgical processes, tens of thousands of tons of metal are produced per week.

Consequently, titanium is very expensive, despite its availability as the fourth most-abundant structural metal in the earth's crust, and its use is limited to highly specialized applications where its superior properties of light weight, high strength, and high corrosion resistance outweigh its cost. About 50,000 tons of titanium are produced annually, compared with 13 million tons of stainless steel.

Many attempts have been made to replace the Kroll process, and even Kroll, in the 1950s, predicted that an electrolytic process would supersede his process within 15 years. Conventional thinking has been that to prepare titanium electrochemically, it is necessary to dissolve a titanium salt in a molten salt, such as sodium chloride or titanium dioxide in a slag phase. When a current is passed between the anode and cathode, titanium metal deposits at the cathode, and chlorine, a severe pollutant, evolves at the anode. Since the temperature is below the melting point of titanium, this process has proved to be exceptionally difficult, as deposition of solid titanium from a molten salt results in a very fine powdery metal that is prone to oxidation. In addition, titanium can exist in several oxidation states in the salt, which can greatly reduce the efficiency of the process.

In the molten calcium chloride process, titanium ions are not formed at any stage, and no chlorine is produced. Furthermore,

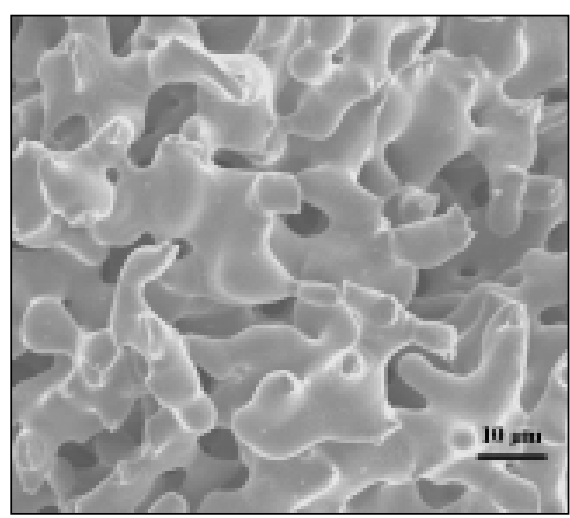

Figure 1. Scanning electron micrograph of electrolytic titanium extracted from solid

titanium dioxide in molten calcium chloride.

as the titanium is not deposited from the salt, it is not in a highly reactive form, but rather has a structure similar to that of the Kroll titanium sponge (see Figure 1). Another unexpected observation was that although titanium dioxide is an insulator, it acts as an efficient cathode: As soon as a minute amount of oxygen is removed, the material becomes an electronic conductor that allows electrochemical processes to take place. In the overall process, an insulating oxide is made into the cathode in an electrochemical cell, and the oxygen is extracted, leaving pure titanium. Another advantage of this technology is that mixtures of oxides can be blended togetherand reduced electrochemically-into alloys. This overcomes many of the problems, such as oxidation and segregation, associated with the alloying of reactive metals. The developers also suggest that their process makes it possible to produce 
alloys that cannot be synthesized using conventional techniques. Furthermore, the product may be suitable for fabrication using powder metallurgical techniques, which eliminate casting, machining, and other costly fabrication processes. Most significant, this process can presumably be applied to the reduction of many metal oxides, particularly those that are difficult and expensive to reduce or where the metals are highly reactive.

\section{Opportunities}

The University of Cambridge is interested in establishing alliances with com- panies that can aid in the exploitation or commercialization of this technology.

Source: Prof. Derek Fray, Professor of Materials Chemistry, Department of Materials Science and Metallurgy, University of Cambridge, Pembroke Street, Cambridge CB2 3QZ, UK; tel. 44-1223-334306; fax 44-1223334567; e-mail djf25@cam.ac.uk.

\section{Direct Nanolithographic Technique Hinges on Oxide-Tip Voltage Difference and Ambient Humidity}

The concept of generating patterned structures directly on a substrate has many advantages compared with traditional indirect lithography techniques that involve multiple-step procedures. The ability to position the probe tip with subnanometer precision has stimulated efforts to use scanning probe microscopes (SPMs) to fabricate nanoscale features on surfaces. Researchers at Lawrence Berkeley National Laboratory (LBNL) have developed a scheme for the nanometer-scale patterning of the surface of the conducting spinel lithium manganese oxide $\left(\mathrm{LiMn}_{2} \mathrm{O}_{4}\right)$. The technique demonstrates that a localized surface chemical change can be confined to a depth that depends on the oxide-tip voltage difference and ambient humidity.

The researchers used an atomic force microscope (AFM) equipped with an electronically conductive tip to both image and modify the surface of a thin film of the conducting oxide $\mathrm{LiMn}_{2} \mathrm{O}_{4}$ in air. In modifying the electronic conductance of $\mathrm{LiMn}_{2} \mathrm{O}_{4}$ at nanometer-scale resolution, the key parameter is the sign of the voltage difference between the AFM tip and the sample. A conductance pattern can be produced with a negatively charged tip and then read with a positively charged tip. In this experimental approach, the AFM probe is used as a preparatory and analytical tool.

A single scan of the AFM tip over the oxide surface simultaneously produces two images: a topographic image and a conductance image; the latter represents oxide-tip current variations during scanning at a given oxide-tip voltage difference. Figure 1 shows (a) a topographic AFM image of a representative $5 \mu \mathrm{m} \times$ $5 \mu \mathrm{m}$ area of a $\mathrm{LiMn}_{2} \mathrm{O}_{4}$ surface and (b) the corresponding conductance image or an oxide-tip current response of the same area with the oxide poised at $-1.0 \mathrm{~V}$ relative to the AFM tip. Prior to the imaging shown in Figure 1, each of five separate
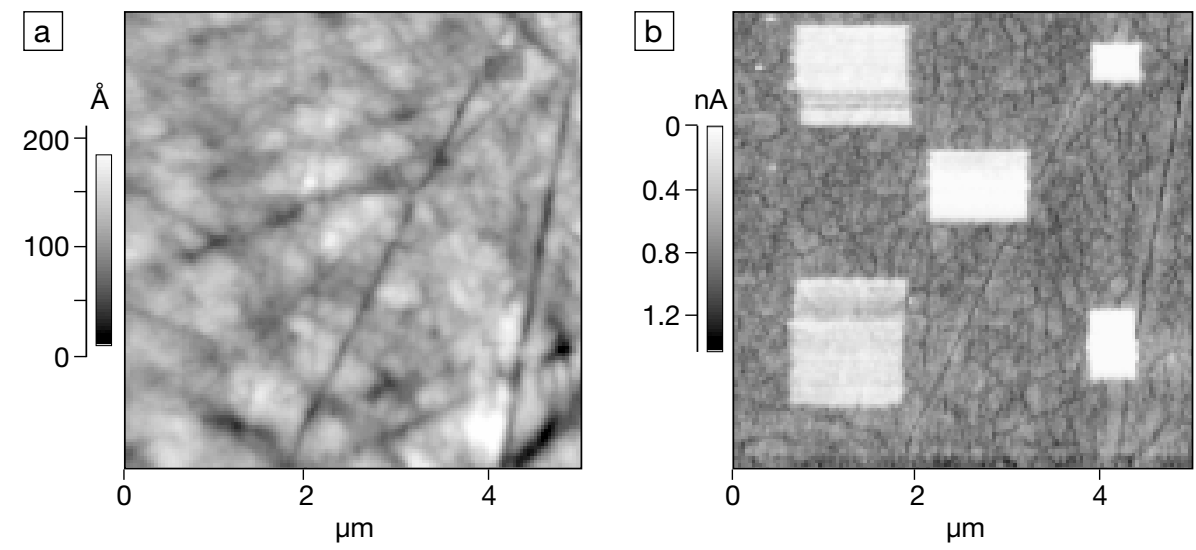

Figure 1. Atomic force microscope (AFM) images of (a) surface topography and $(b)$ conductance of a LiMn ${ }_{2} \mathrm{O}_{4}$ surface $(5 \mu \mathrm{m} \times 5 \mu \mathrm{m})$ at ambient conditions recorded with the oxide poised at $-1.0 \mathrm{~V}$ versus the AFM tip, after surface modification of five areas produced at the opposite oxide-tip polarity.

areas within the same $5 \mu \mathrm{m} \times 5 \mu \mathrm{m}$ region were pre-scanned one time with the oxide poised at $+1.0 \mathrm{~V}$ relative to the AFM tip. The white rectangular regions on the conductance image correspond to the pre-scanned areas.

The conductance was substantially reduced in areas pre-scanned with a negatively charged AFM tip. Unexpectedly, there is no visible change in the oxide surface morphology that accompanies changes in the film conductance. Compared to imaging with a positively charged AFM tip, a negatively charged AFM tip caused a rapid decline of film conductance. By reducing the oxide-tip voltage difference to less than $-0.1 \mathrm{~V}$, the researchers could further suppress the resistance buildup while preserving good contrast of the conductance image. These findings demonstrate "write" and "read" modes of the same AFM probe at the $\mathrm{LiMn}_{2} \mathrm{O}_{4}$ surface. The as-produced pattern can be erased by scanning over the entire region with a negatively charged AFM tip.

A thin layer of ambient adsorbed water plays a key role in the electrochemical mechanism of the modification process. Such a thin layer of adsorbed water exists on most materials under ambient conditions. The extent of localized chemical change can be controlled by a combination of the oxide-tip voltage difference and the ambient humidity level. Experiments that were carried out at $100 \%$ relative humidity led to localized etching of the $\mathrm{LiMn}_{2} \mathrm{O}_{4}$ substrate and produced wells and trenches several tens of nanometers deep.

Applications for this nanolithographic technique are anticipated in the semiconductor industry to produce active and/or passive elements in integrated circuits, such as single-electron transistors and microbatteries for memory chips, and also as an encrypting tool to save information at high density.

\section{Opportunities}

LBNL is seeking partners for licensing and/or collaborative development and commercialization of this technology.

Source: Robert Kostecki, Lawrence Berkeley National Laboratory, MS 90-1070, 1 Cyclotron Road, Berkeley, CA 94720, USA; tel. 510-4866002; fax 510-486-4995; e-mail r_kostecki@lbl. gov; or Technology Transfer Department, tel. 510-486-6467; fax 510-486-6457; e-mail TTD@lbl. gov; www. lbl.gov/Tech-Transfer. 